\title{
Las energías renovables en la actividad turística. Innovaciones hacia la sostenibilidad
}

\section{Renewable energy and the tourism sector. Innovations towards sustainability}

\author{
Xavier Lastra Bravoㄹ, Juan Gabriel Coloma Martínez ${ }^{1}$, Dennise Espinosa Jarrín¹, Fernando Herrera Ronquillo ${ }^{1}$ \\ ${ }^{I}$ Universidad Central del Ecuador. Facultad de Ciencias Agrícolas. Carrera de Turismo Ecológico. Jerónimo Leiton y Av. La Gasca s/n. Ciudadela \\ Universitaria. Quito. 170521. Ecuador
}

\begin{abstract}
Resumen
El consumo de combustibles fósiles ha provocado graves impactos ambientales a nivel mundial, principalmente por la emisión de gases de efecto invernadero (GEI), causa del cambio climático. Por ello, en las últimas décadas se ha dado un mayor impulso a las energías limpias o procedentes de fuentes renovables, caracterizadas principalmente por su menor emisión de GEI, el aumento directo en el ahorro económico y en la reducción de los problemas de suministro y almacenamiento de combustible. El turismo, por su cada vez mayor importancia en la economía nacional, representa un sector con grandes posibilidades para implementar este tipo de tecnologías limpias, así como también de implementar medidas para un mayor ahorro energético que conlleva a una mayor eficiencia energética. En este artículo, se analiza las posibilidades de uso de energía procedente de distintas fuentes renovables en el sector turismo. Primero, se analiza la importancia de las energías renovables en la actualidad. Posteriormente, se aborda brevemente la situación del turismo en el Ecuador de acuerdo con las estadísticas oficiales. Finalmente, se recoge información bibliográfica sobre las distintas fuentes de energía renovable que son aplicables en el sector turismo en el Ecuador, y medidas para mejorar la eficiencia energética de sus instalaciones.
\end{abstract}

Palabras clave: turismo, energías limpias, eficiencia energética, Ecuador.

\begin{abstract}
Fossil fuels consumption has caused severe environmental impacts worldwide, mainly by the greenhouse gasses emissions which have led into of climate change. Therefore, the latest decades clean energies of renewable energy resources have been boosted. These energies are characterized by lower greenhouse gasses emissions, direct increase in the cost savings and problems supply and fuel storage reduction. By its growing importance in the national economy, Tourism represents a sector with great potential for implementing clean technologies as well as energy savings measures that leads to greater energy efficiency. In this manuscript, the possibilities of renewable energy use from several sources in the tourism sector are analysed. First, the renewable energy prominence at present is analysed. Then the main figures of tourism in Ecuador are briefly shown. Finally, bibliographic information on the different renewable energy sources that could be applied in the tourism sector in Ecuador and the measures to improve the energy efficiency of their facilities is summarized.
\end{abstract}

Keywords: tourism, clean energy, energy efficiency, Ecuador. 


\section{Introducción}

El alto consumo de energía procedente de combustibles fósiles constituye una gran preocupación mundial, junto con las fuentes limitadas de los mismos, el cambio climático y el calentamiento global y sus posibles consecuencias a largo plazo, y el crecimiento de la población (Vera \& Langlois, 2007; Omer, 2008a; Schreyer \& Mez, 2008). Más aún, cuando la energía es el principal insumo para el desarrollo socio-económico, dependiendo las áreas urbanas dependen de las fuentes de energía comercial y las rurales de las no comerciales (e.g. leña y residuos agrícolas), por tanto, su sostenibilidad es un factor importante a considerar (Omer, 2008a). El desarrollo de un territorio, generalmente se traduce en una mejor calidad de vida, y por lo tanto, en un mayor consumo de energía primaria en todos los sectores: transporte, industria, servicios, residencial, etc. (Abulfotuh, 2007). La fuerte relación existente entre el uso de energía y la actividad económica, según la teoría económica, está dada porque la energía, junto con el capital y la mano de obra, es un factor de entrada en la función de producción, y por tanto uno de los principales motores del crecimiento económico (Kemmler \& Spreng, 2007).

En concreto, el calentamiento global, o efecto invernadero, es consecuencia del aumento de las concentraciones de dióxido de carbono $\left(\mathrm{CO}_{2}\right)$, metano $\left(\mathrm{CH}_{4}\right)$, cluoroflurocarbonados, halones, óxido nitroso $\left(\mathrm{N}_{2} \mathrm{O}\right)$, ozono y peroxiacetilnitrato en la atmósfera, que a su vez incrementan la manera en cómo estos gases atrapan el calor irradiado por la superficie terrestre, elevando la temperatura de la tierra (Dincer \& Rosen, 1999). La emisión y acumulación de los gases de efecto invernadero (GEI) en la atmósfera es resultado directo del progreso y desarrollo tecnológico mundial (Omer, 2008a; Sari \& Soytas, 2009), y probablemente es el problema ambiental más importante causado por las actividades relacionadas con la energía (Dincer \& Rosen, 1999; EC, 2009).

Las políticas de eficiencia energética son un aspecto importante en la consecución del desarro- llo sostenible y en la reducción de las emisiones de GEI y el cambio climático, poniendo de manifiesto la interrogante sobre cómo lograr que estas políticas sean lo más efectivas posibles (Varone \& Aebischer, 2001). Los países que han incrementaron su eficiencia energética, a través de la ejecución de políticas y programas, han tenido éxito en reducir el consumo de energía, su intensidad energética, y sus emisiones de GEI. Además, los impactos positivos de carácter ambiental (reducción del consumo de recursos, de la contaminación, degradación de ambientes, etc.) y social (p.e. la generación de empleos directos e indirectos, redistribución de riqueza e ingresos de divisas, la revalorización de la cultura local, etc.).

En este sentido, se han llevado a cabo estudios para medir la relación entre la reducción de emisiones de GEI y el crecimiento económico. Por ejemplo, se ha establecido que a largo plazo no existe una relación de equilibrio entre el consumo de energía, el trabajo y los ingresos (Sari \& Soytas, 2009), por tanto, la reducción en el consumo de energía, al no perjudicar su crecimiento económico, puede constituirse en una herramienta eficaz para reducir las emisiones de $\mathrm{CO}_{2}$. También, se ha determinado que existe una relación no linear estadísticamente significativa entre emisiones de $\mathrm{CO}_{2}$ e ingresos, y una relación positiva entre consumo eléctrico y emisiones de $\mathrm{CO}_{2}$, en cinco países de la Asociación de Naciones del Sudeste Asiático (Lean \& Smyth, 2010).

Esta situación requiere el uso de fuentes energéticas más limpias y seguras que permitan alcanzar una mayor sostenibilidad (Dincer \& Rosen, 1998; Lidula et al., 2007). El uso de energía procedente de fuentes renovables está íntimamente ligado al desarrollo sostenible, ya que para alcanzarlo y obtener avances ante los problemas ambientales existentes, la sociedad precisa de un suministro sostenible de recursos energéticos y de un uso eficaz y eficiente de los mismos (Omer, 2008a). En este sentido, se define energía sostenible cuya producción y consumo tenga un mínimo impacto en la salud humana y en el funcionamien- 
to saludable de los sistemas ecológicos vitales, incluido el ambiente en general (Omer, 2008b). Para Charters (2001), la energía procedente de fuentes renovables es un recurso sostenible y disponible a largo plazo, de manera sencilla y duradera, obtenida a un costo razonable y que puede ser utilizado en todas las tareas sin causar impactos negativos. Pero, su producción presenta algunos impactos negativos que deben ser resueltos para alcanzar una visión equilibrada de sus virtudes y deficiencias (Abbasi \& Abbasi, 2000).

En este artículo, se analiza las posibilidades de uso de energía procedente de distintas fuentes renovables en el sector turismo. Primero se recoge brevemente la situación del turismo en el Ecuador de acuerdo con las estadísticas oficiales. Posteriormente, se recoge información bibliográfica sobre las distintas fuentes de energía renovable que son aplicables en el sector turismo en el Ecuador, y medidas para mejorar la eficiencia energética de sus instalaciones.

\section{Importancia del sector turismo en cifras}

En Ecuador, el Turismo fue en el año 2013 el cuarto sector por ingresos de divisas, con un monto total de USD 1,251.3 millones (5\% del total), después de los ingresos percibidos por banano, camarón y productos del mar. En relación con el PIB, representó $1.3 \%$, con un incremento de 2 puntos porcentuales en comparación con el 2009 (MINTUR, 2014).

La recaudación tributaria de hoteles y restaurantes de carácter turístico alcanzó los USD 112,564,164 (MINTUR, 2014). Se observó un incremento superior al 50\% en comparación con el 2009. Por provincias, Pichincha aportó con el 55.9\%, seguida de Guayas (22\%) y Azuay (5.1\%). Procedente de las agencias de viajes, organizadores de excursiones y guías turísticos se recaudaron 19,321,138 USD, un 87.6\% más en comparación con 2009 .

El número de establecimientos turísticos registrados fue de 21,069 (2013), incluyendo alo- jamiento $(4,672)$, servicio de alimentos y bebidas $(14,057)$, transporte (378), operación (734), intermediación $(1,034)$ y otras instalaciones recreativas (194) (MINTUR, 2014). Estos establecimientos generaron 114,113 empleos directos, principalmente en alojamientos $(31,904)$ y servicios de alimentos y bebidas $(69,782)$. El número total de plazas fue de 206,384 .

\section{Energías renovables y turismo}

El consumo de energía procedente de fuentes limpias tiene entre sus ventajas la reducción del consumo de combustibles fósiles, que conlleva a su vez un aumento directo en el ahorro económico y en la reducción de los problemas de suministro y almacenamiento de combustible (EC, 2014). Además, se minimiza la producción de contaminantes como los procedentes de la quema de combustibles fósiles, la energía producida es de baja tensión, reduciendo las probabilidades de accidentes peligrosos, y contribuyen a la descentralización y diversificación de la producción de energía. En relación con el empleo, se generan más puestos de trabajo comparado con la producción convencional de energía. Finalmente, promueven las economías rurales, donde no es imprescindible el tendido eléctrico para la distribución de energía, ya que ésta puede ser suministrada por energías limpias. Para el sector hotelero, el uso de las energías limpias podría dar un mayor impulso al turismo rural, especialmente a las pequeñas instalaciones que podrían ser autosuficientes energéticamente. En la Tabla 1 se recogen las principales ventajas y desventajas de las distintas fuentes de energía renovable utilizadas en la actualidad en el sector turístico. 
Tabla 1. Ventajas y desventajas de los tipos de energía procedente de fuentes renovables.

\begin{tabular}{|c|c|c|}
\hline & Ventajas & Desventajas \\
\hline Termosolar & $\begin{array}{l}\text { - bajo costo } \\
\text { - contrastado y fiable } \\
\text { - amplia disponibilidad } \\
\text { - se puede instalar en cualquier zona (no re- } \\
\text { quiere red eléctrica) }\end{array}$ & $\begin{array}{l}\text { - estacionalidad } \\
\text { - requiere sistemas de almacenamiento térmico para pe- } \\
\text { riodos nublados o ser complementado con otras fuentes } \\
\text { - requiere una inversión significativa }\end{array}$ \\
\hline Fotovoltaica & $\begin{array}{l}\text { - se puede generar energía incluso en días nu- } \\
\text { blados } \\
\text { - no produce ruido, emisiones nocivas o gases } \\
\text { contaminantes } \\
\text { - puede instalarse tanto en zonas rurales como } \\
\text { urbanas } \\
\text { - requieren un mantenimiento mínimo } \\
\text { - se pueden instalar módulos fácilmente y am- } \\
\text { pliarse cuando se requiera }\end{array}$ & $\begin{array}{l}\text { - tienen una eficiencia de conversión relativamente baja } \\
(10-15 \%) \text {. Sin embargo, la eficiencia aumenta conforme } \\
\text { se realizan mayores avances científicos } \\
\text { - requieren superficie para su instalación }\end{array}$ \\
\hline Geotermal & $\begin{array}{l}\text { - alta disponibilidad } \\
\text { - muy bajo impacto visual } \\
\text { - no depende de las condiciones climáticas } \\
\text { - durabilidad a largo plazo de la instalación }\end{array}$ & - requieren altas inversiones \\
\hline Biomasa & $\begin{array}{l}\text { - bajo costo } \\
\text { - puede ser almacenado y utilizado } \\
\text { - libera menos CO que los combustibles fósiles }\end{array}$ & $\begin{array}{l}\text { - la quema de residuos sólidos urbanos (basura) puede li- } \\
\text { berar sustancias químicas } \\
\text { - posible presencia de metales pesados en las cenizas pro- } \\
\text { ducto de la combustión } \\
\text { - los cultivos energéticos requieren gran cantidad de terreno } \\
\text { - afecta el paisaje agrícola, pudiendo producir riesgo de } \\
\text { reducción de la diversidad biológica y alto consumo de } \\
\text { fertilizantes y pesticidas }\end{array}$ \\
\hline Eólica & $\begin{array}{l}\text { - bajo costo } \\
\text { - instalación rápida } \\
\text { - proporciona una alta potencia } \\
\text { - contaminación ambiental baja }\end{array}$ & $\begin{array}{l}\text { - ruido (mecánico y aerodinámico) } \\
\text { - puede causar interferencia electromagnética } \\
\text { - impacto visual } \\
\text { - impacto ambiental si interfiere con rutas migratorias de aves } \\
\text { - produce sombra que puede afectar a la fauna }\end{array}$ \\
\hline
\end{tabular}

Fuente: UNEP (2003), Hotel Energy Solutions (2011), EC (2014)

\subsection{Sistemas termosolares}

Estos sistemas pueden proveer calor o agua caliente sanitaria (ACS) de manera más rentable en instalaciones que requieren de temperaturas inferiores a $100^{\circ} \mathrm{C}$, tanto en instalaciones domésticas pequeñas como en sistemas industriales grandes.

En la actualidad, los calentadores solares de agua son sistemas maduros y comerciales, utilizados ampliamente en negocios de hostelería que requieren de un suministro constante de ACS (UNEP, 2003). En las empresas de hostelería, aproximadamente el 12\% de los costos totales de la energía y el $20 \%$ del consu- mo de energía corresponden a ACS, porcentajes que podrían ser reducidos mediante el uso de los calentadores solares. De esta manera, se reduciría el pago de la factura de la luz y de combustibles (principalmente gas de uso doméstico), reduciría el riesgo financiero por cambios en el precio de la energía y compensaría un deficiente abastecimiento. En los climas subtropicales y tropicales, entre el 80 y el $100 \%$ de las necesidades de ACS pueden ser cubiertas, especialmente en las zonas ecuatoriales y tropicales, donde la disponibilidad de sol es abundante a lo largo del año (UNEP, 2003; EC, 2014). Además, considerando una duración media de 20 años y el bajo mantenimiento que requieren, son una opción rentable. Más aún, cuando en la 
zona ecuatorial se estima que con dos horas de luz solar brillante sobre un panel colector de aproximadamente $2 \mathrm{~m}^{2}$, se podría mantener el agua de un tanque de 225 litros entre 40 y $60^{\circ} \mathrm{C}$, cuando se utiliza a una tasa de consumo continua de 8.8 litros $\mathrm{min}^{-1}$.

La mayoría de los calentadores solares incluyen dispositivos de calefacción adicional para proporcionar ACS cuando la luz solar es ineficiente (en la noche o en periodos nublados) o cuando se requiere una mayor temperatura (mayor a $45-55^{\circ} \mathrm{C}$ ). Pueden ser adaptados a los edificios existentes con algunas modificaciones, y en los nuevos se integran en su diseño (instalándolos generalmente en el techo). En su funcionamiento posee varias ventajas, como el uso de menores tramos de tubería y un mayor acceso a las mismas, lo que ayuda a reducir los costos.

Para su uso en calefacción, se distribuyen adecuadamente las tuberías de ACS en todo el edificio, conectadas a radiadores o colectores especiales. El principal uso en este sentido es para el calentamiento de piscinas, siendo una opción simple y rentable. Los colectores solares para este uso suelen ser de mayor tamaño que aquellos utilizados para ACS. Tales sistemas incorporan generalmente una manta plástica aislante sobre la piscina para evitar la pérdida de calor cuando no está en uso. Se estima que estos sistemas ayudan a sustituir el consumo de $120 \mathrm{MWh}$ o 12,000 $\mathrm{m}^{3}$ de gas, $\mathrm{y}$ a reducir la emisión de $65 \mathrm{~kg}$ de $\mathrm{N}_{2} \mathrm{O}$ y 32 $000 \mathrm{~kg}$ año ${ }^{-1}$ de $\mathrm{CO}_{2}$ (UNEP, 2003; EC, 2014).

La cocción a través de cocinas solares puede ser aplicada de manera generalizada en actividades de senderismo y turismo rural en general, evitando el uso de leña o carbón.

\subsection{Energía fotovoltaica}

La generación de electricidad en áreas remotas puede realizarse a través de energía fotovoltaica, supliendo de esta manera los problemas de suministro por parte de la red eléctrica general (UNEP, 2003). Su plazo de amortización es más largo, en relación con otras fuentes de energía renovable (EC, 2014). Estos sistemas generalmente se utilizan junto con otras fuentes de energía (por ejemplo generados a diésel). Los paneles solares son unidades de pequeña superficies que pueden ser instalados, principalmente, en los techos de los edificios. En los países desarrollados existe la posibili- dad de vender el exceso de energía a las empresas energéticas, de manera que se pudiera obtener un ingreso extra. En relación con la estética de los edificios, factor que tiene gran importancia en las actividades turísticas, en la actualidad se busca incorporar los paneles solares en el diseño del edificio, de manera que se mimeticen con la estructura turística.

Además de la producción de energía eléctrica, otros usos en la actividad turística son purificación de agua, desalinización, alimentación de equipos pequeños como parquímetros, letreros o cámaras de video (EC, 2014).

\subsection{Energía geotérmica}

La energía geotérmica, procedente del calor de la Tierra, se puede recuperar a temperaturas comprendidas entre 35 y $150^{\circ} \mathrm{C}$ (UNEP, 2003). Se utiliza para calefacción de edificios y de invernaderos, en instalaciones de acuicultura, para proporcionar calor en procesos industriales y producción de electricidad (a altas temperaturas). La eficiencia en el uso de la energía geotérmica de manera directa es del 50-70\%, en comparación con el 5-20\% de su uso indirecto para generar electricidad, aunque el calor residual de la generación de electricidad también se puede utilizar, mejorando la eficiencia global del sistema (UNEP, 2003).

El principal uso comercial de este sistema son las bombas de calor geotérmicas (BCG), que aprovechan el calor, casi constante y natural, de la tierra. Se utilizan para calefacción y refrigeración de edificios, ACS y para piscinas. Si bien su instalación es más costosa, se amortiza a corto plazo ya que es uno de los sistemas más eficientes y rentables. Utiliza mucho menos energía que los sistemas convencionales de calefacción/refrigeración, y provee una cantidad de energía 3 a 4 veces mayor que la que consume. Esto se debe a que las BCG mueven el calor hacia o desde el suelo en lugar de generarla mediante bombas de calefacción o compresores eléctricos. En Norteamérica se ha utilizado esta tecnología en hoteles, reportando un ahorro energético del $40-70 \%$ en el invierno, y $30-60 \%$ en verano (UNEP, 2003). En su mayoría se han utilizado para la refrigeración de locales, y se han diseñado para cubrir toda la demanda de energía para aire acondicionado de los edificios. Otras ventajas son su funcionamiento silencioso, la mejora de la 
calidad del aire interior (incluyendo niveles confortables de humedad, y la reducción de contaminantes y de los alérgenos por la falta de combustión), ausencia de dependencia de aire desde el exterior del edificio, y la ausencia de instalaciones externas (por ejemplo, torres de enfriamiento y bombas de aire) que podrían poner en peligro la estética exterior.

Para calefacción, el calor de las aguas subterráneas es absorbido por el refrigerante que circula en las BCG, el cual una vez caliente es bombeado hacia una bomba de calor que transfiere el calor del refrigerante al agua. Cubre aproximadamente el $80 \%$ de la demanda. El agua caliente se puede utilizar directamente como ACS o circular a través de radiadores para la calefacción de los edificios. También, se puede hacer circular aire alrededor de las tuberías de agua caliente, para calentarlo, a través de ductos de aire acondicionado, lo que se conoce como un sistema de agua-aire. Para refrigeración, el proceso anterior se invierte, es decir, el refrigerante absorbe el calor de los espacios interiores del edificio y lo transfiere al suelo.

\subsection{Biomasa}

La biomasa puede ser aprovechada en varias aplicaciones de calefacción, en función del tipo y disponibilidad de recursos (UNEP, 2003). Los principales recursos de biomasa sostenible son los residuos industriales y agrícolas (bagazo de la caña de azúcar, residuos madereros, y residuos de cultivos de ciclo corto), residuos orgánicos (ganadería, paja), cultivos energéticos (caña de azúcar, maíz, plantaciones forestales de rotación corta), residuos domésticos y municipales (aguas residuales y basura).

Los principales procesos para la utilización de las diversas fuentes de biomasa incluyen la combustión directa (en calderas o como combustibles en motores), gasificación (conversión física o química a combustible gaseoso secundario), digestión anaeróbica bacteriana (producción de biogás rico en metano) y conversión bioquímica (producción de etanol, metanol u otros combustibles líquidos).

De las distintas fuentes, procesos y tecnologías para aprovechar la biomasa, la combustión directa es el proceso comercial más desarrollado, especialmente de madera (pellets). En muchas zonas rurales, donde la deforestación no es un problema, la leña es un combustible más barato que el gas o la electricidad, incluso considerando los costos de mano de obra para el corte, recolección y reabastecimiento.

Desde el punto de vista turístico, hay que considerar que los turistas suelen apreciar la estética de una fogata o chimenea, especialmente en las cabañas o en las habitaciones de hotel, aunque requiere mayor mano de obra para abastecerlas. Para las empresas turísticas localizadas cerca de aserraderos $\mathrm{u}$ otras plantas de procesamiento de madera, el uso de aserrín y otros residuos madereros podría ser una buena opción para la calefacción y/o cocción.

\subsection{Energía eólica}

La generación de energía a partir del viento es una tecnología madura y económicamente competitiva en comparación con la mayoría de combustibles fósiles (UNEP, 2003). El uso de turbinas eólicas es una opción atractiva para las instalaciones turísticas localizadas en zonas costeras, en planicies y en puertos de montaña expuestas a un flujo constante de viento. Las instalaciones requieren poco mantenimiento, y no emiten GEI u otro tipo de polución.

Para su instalación se requiere analizar la velocidad del viento de la zona, factor crítico de este tipo de instalaciones, la cual debe ser de al menos 5.5-6 $\mathrm{m} \mathrm{s}^{-1}$ o $22 \mathrm{~km} \mathrm{~h}^{-1}$, para que sea viable comercialmente. Considerando que la velocidad del viento es intermitente, se recomienda instalar estas facilidades en zonas donde previamente se ha realizado un estudio de las corrientes y la velocidad del viento (EC, 2014).

De acuerdo con los requerimientos energéticos, se pueden instalar facilidades pequeñas $(<1 \mathrm{KW})$, medianas (100-700 KW) y grandes $(>700 \mathrm{KW})$. En el caso del sector turismo, facilidades pequeñas y medianas son las más apropiadas. Es importante que previo a su instalación se consideren los impactos ambientales que pueden producir, entre ellos el impacto sobre el paisaje, el ruido, la aviación y los recorridos migratorios de las aves, entre otros. En zonas rurales también puede ser una manera de alcanzar autosuficiencia energética, principalmente cuando se combina con energía fotovoltaica (sistemas híbridos), u otros generadores. 
En el caso de los sistemas híbridos, el pico máximo de producción de la energía eólica no coincide con el de la producción de energía fotovoltaica, compensándose. Es decir, durante el día la energía fotovoltaica alcanza su pico de producción, y generalmente los vientos suelen ser de menor velocidad, mientras que por la noche, los vientos suelen ser más fuertes, aumentando la producción de energía eólica, y obviamente reduciéndose la producción de energía fotovoltaica. Cuando se den circunstancias adversas, no hay viento y es de noche, los sistemas híbridos incluyen generadores en base a combustibles fósiles para compensar la falta de producción de energía renovable. Otra aplicación útil de esta tecnología es para el bombeo de agua, tanto para la agricultura como para el turismo rural.

\subsection{Medidas de eficiencia energética de aplicación a corto plazo}

La energía que se consume en el sector hotelero no solo es costosa, sino que también contribuye a los problemas ambientales. Los hoteles se encuentran entre los cinco principales tipos de edificaciones que consumen energía, después de las instalaciones para la producción de alimentos, comercio y salud. Un hotel promedio libera anualmente entre $160-200 \mathrm{~kg}$ de $\mathrm{CO}_{2}$ por $\mathrm{m}^{2}$ de superficie de habitación. Al reducir las emisiones de $\mathrm{CO}_{2}$, los hoteles pueden hacer una contribución positiva al medio ambiente $\mathrm{y}$, al mismo tiempo, reducir sus costos operativos (Hotel Energy Solutions, 2011).

Como se mencionó anteriormente, el sector hotelero podría ahorrar en costos operativos mediante el uso de energía procedente de fuentes renovables, así como también mediante la aplicación de medidas de eficiencia energética. Se estima que el $40 \%$ de la energía utilizada en los hoteles es electricidad, y el 60\% proviene de combustibles fósiles. Las tres cuartas partes se utilizan para calefacción, ACS, aire acondicionado y ventilación, e iluminación. Por tanto, estos usos de la energía presentan un gran potencial para aumentar la eficiencia energética (Hotel Energy Solutions, 2011).

Existen distintas medidas que pueden ser aplicadas por el sector turismo para reducir el consumo de energía en sus instalaciones, entre esas medidas se encuentran:

- Modificaciones en la iluminación. Posiblemente es el método más asequible y práctico para mejorar la eficiencia energética de las instalaciones turísticas, a través del uso de focos fluorescentes compactos, de bajo consumo, y de sensores para la iluminación de áreas menos transitadas. Otro método utilizado es un mayor aprovechamiento de la iluminación natural.

- Restricción del consumo energético en las habitaciones. Mediante el uso de tarjetas para limitar automáticamente el consumo de electricidad en las habitaciones cuando el turista no se encuentra en ellas, evitando el consumo inútil de energía.

- Electrodomésticos energéticamente eficientes. En la actualidad los electrodomésticos poseen una certificación energética en función de su eficiencia, consumiendo menos energía, y por tanto, ahorrando en costos de funcionamiento. Los principales electrodomésticos utilizados en el sector turismo son refrigeradores, congeladores, lavavajillas y lavadoras de carga frontal.

- Regular la calefacción y la refrigeración de espacios. Generalmente es la actividad que más consume energía en una instalación hotelera, por ello, es importante, además de incorporar equipos más eficientes energéticamente, regular la temperatura de acuerdo a las necesidades reales y el grado de ocupación de cada área, habitaciones, espacios comunales, zonas de ocio, etc.

- La compensación de carbono. Al igual que en otros sectores de la industria del turismo, muchos alojamientos ofrecen programas de compensación de carbono que permiten a los turistas compensar el impacto de su viaje.

- Compra de créditos de energía renovable. Si el alojamiento consume energía procedente de fuentes renovables (élica, solar o hidroeléctrica), se da un paso más hacia la sostenibilidad.

Es importante que el personal también participe y sea consciente de la importancia de su actuación para alcanzar una mayor eficiencia energética. Su mayor conocimiento, grado de concientización y partici- 
pación activa, serán determinantes para lograr que el turista también adquiera mejores prácticas en el uso de las instalaciones turísticas. El transmitir que las restricciones en el uso de los recursos no son para incomodar o reducir el confort de los turistas, sino para aumentar el cuidado del ambiente, será una tarea fundamental del personal de las instalaciones turísticas.

En cuanto al diseño, el considerar el diseño pasivo de edificios, que busca mantener un equilibrio entre la economía y la construcción, es una buena opción para reducir el consumo de energía. Para ello, se requiere considerar una buena envolvente, un buen sistema de aislamiento y realizar un control riguroso de las infiltraciones de aire indeseadas y de los puentes térmicos. El diseño solar pasivo es una de las formas más simples y rentables para calentar y enfriar edificios, estimándose un ahorro de hasta el $40 \%$ en los costos de calefacción (UNEP, 2003). El diseño pasivo se centra en reducir al máximo la demanda energética, su principal valor será la rigurosidad de su diseño, el cálculo de su proyecto y la ejecución de la obra, garantizando que los valores teóricos calculados se ajusten a los valores reales obtenidos una vez construido el edificio (IDAE, 2011). Se ha comprobado que las medidas más eficientes son la disposición de aislamiento térmico en envolventes, la instalación de bombillas de bajo consumo, la instalación de colectores termosolares para la producción de ACS, equipos de instalación de energías renovables y el mantenimiento regular de los sistemas de calefacción (Gaglia et al, 2006).

A través del uso de algunos principios básicos se puede aprovechar la variación diaria y estacional

\section{Referencias}

Abbasi, S.A \& Abbasi. N. (2000) The likely adverse environmental impacts of renewable energy sources. Applied Energy, 65, 121-144.

Abulfotuh, F. (2007) Energy efficiency and renewable technologies: the way to sustainable energy future, Desalination, 209, 275-282.

Charters, W.W.S. (2001). Developing markets for renewable energy technologies, Renewable Energy, 22, 217-222.

Dincer, I., \& Rosen, M.A. (1998). $A$ worldwide perspective on energy, environment and sustainable del sol. En climas más cálidos, la construcción de edificios con aleros o salientes más amplios, con porches (espacios arquitectónicos abiertos lateralmente y cerrados por la parte superior adosado a una construcción), el uso en los techos de colores claros y de materiales/diseños que no absorben el calor y promueven la ventilación cruzada pueden ayudar a evitar o reducir la necesidad de sistemas de aire acondicionado más costosos. En los edificios nuevos, la inclusión de sistemas pasivos implica un costo adicional muy bajo o casi nulo, ya que simplemente se aprovecha la orientación y el diseño de un edificio para capturar y utilizar la radiación solar. Los edificios solares pasivos se ven como cualquier otro edificio, pero son generalmente más cómodos para vivir y menos costosos para funcionar.

\section{Conclusiones}

En la actualidad se dispone de una gran variedad de energía procedente de fuentes renovables, las cuales pueden ser incorporadas en las instalaciones turísticas en función de su disponibilidad en cada territorio.

La energía fotovoltaica y termosolar son las tecnologías más utilizadas, cuyos beneficios, tanto económicos como ambientales, están ampliamente demostrados. En el caso de Ecuador, existe una alta potencialidad de uso de las distintas fuentes de energía renovable, requiriéndose mayores incentivos gubernamentales para la instalación de pequeñas facilidades en el sector turístico.

development. International Journal of Energy Research, 22(15), 1305-1321.

Dincer, I., \& Rosen, M.A. (1999). Energy, environment and sustainable development. Applied Energy, 64, 427-440.

European Commission (EC) \& Tourism Business Portal. (2014) Which renewable energy sources are the most appropriate for the tourism sector?.

European Communities (EC). (2009) Panorama of energy. Energy statistics to support EU policies and solutions. EUROSTAT, Statistical books. Luxem- 
bourg: Office for Official Publications of the European Communities.

Gaglia, A.G.; Balaras, C.A.; Mirasgedis, S.; Georgopoulou, E.; Sarafidis, Y. \& Lalas. D.P. (2007) Empirical assessment of the Hellenic non-residential building stock, energy consumption, emissions and potential energy savings. Energy conversion and Management, 48, $1160-1175$.

Hotel Energy Solutions (2011) Hotel Energy Solutions: Fostering innovation to fight climate change - Public Report. Hotel Energy Solutions project publications.

Instituto para la Diversificación \& Ahorro de la Energía (IDEA) (2011). Guía práctica de la energía: Consumo eficiente y responsable. España: Gráficas Monterreina, S.A.

Lean, H.H. \& Smyth, R. (2010). CO2 emissions, electricity consumption and output in ASEAN. Applied Energy, 87, 1858-1864.

Kemmler, A. \& Spreng, D. (2007) Energy indicators for tracking sustainability in developing countries. Energy Policy, 35, 2466-2480.

Lidula, N.W.A.; Mithulananthan, N.; Ongsakul, W.; Widjaya, C. \& Henson, R. (2007) ASEAN towards clean and sustainable energy: Potentials, utilization and barriers. Renewable Energy, 32, 1441-1452.
Ministerio de Turismo. (2014). Boletín de Estadísticas Turísticas 2009-2013. Ecuador: Ministerio de Turismo.

Omer, A.M. (2008a). Energy, environment and sustainable development. Renewable and Sustainable Energy Reviews, 12, 2265-2300.

Omer, A.M. (2008b) Green energies and the environment. Renewable and Sustainable Energy Reviews, 12, 1789-1821.

Sari, R. \& Soytas, U. (2009). Are global warming and economic growth compatible? Evidence from five OPEC countries?. Applied Energy, 86, 1887-1893

Schreyer, M. \& Mez, L. (2008). ERENE - European Community for Renewable Energy. Heinrich-Böll-Stiftung, Vol. 3.

United Nations Environment Programme (UNEP). (2003). Switched on. Renewable Energy Opportunities in the Tourism Industry. Division of Technology, Industry and Economics Production and Consumption Branch.

Varone, F. \& Aebischer, B. (2001). Energy efficiency: the challenges of policy design. Energy Policy, 29, 615-629.

Vera, I. \& Langlois, L. (2007). Energy indicators for sustainable development. Energy, 32, 875882. 